\title{
The inhibitor miR-21 regulates macrophage polarization in an experimental model of chronic obstructive pulmonary disease
}

\author{
JunJuan Lu', LiHua Xie', ShengHua Sun ${ }^{1}$
}

\begin{abstract}
INTRODUCTION In chronic obstructive pulmonary disease (COPD), macrophages play an indispensable role. In the lung tissues of COPD patients and smokers, macrophages can be observed to polarize towards M2 phenotype. The molecular mechanism of this process is unclear, and it has not been fully elucidated in COPD. METHODS We bought laboratory animals [C57BL/6 and miR-21 ${ }^{-/-} \mathrm{C} 57 \mathrm{BL} / 6(\mathrm{~F} 1)$ ] from the Jackson Laboratory. The model of COPD mice was established by cigarette smoke (CS) exposure combined with intraperitoneal injection of cigarette smoke extract (CSE). RT-PCR detected the expression levels of inflammatory factors and markers associated with M1 and M2 macrophages. The ratio of M2 macrophages to M1 macrophages was detected by immunohistochemical staining. RESULTS The level of miR-21 was increased in RAW264.7 cells intervened by $\mathrm{CSE}$ and in lung tissue and bone marrow-derived macrophages (BMDMs) from COPD mice. CSE can gradually over time increase the level of miR-21. The proportion of M2 macrophages to M1 macrophages had a positive correlation with miR-21. Knockdowning miR-21 can reduce lung tissue damage. CSE also increased the levels of related inflammatory factors and markers associated with M2 macrophages, and an miR-21 inhibitor can reverse this conversion.

CONCLUSIONS We confirmed that CSE can lead to macrophage transformation to the M2 phenotype and an increase in the expression level of miR-21. Knockdown of the miR-21 gene could inhibit the transformation of macrophages to the M2 phenotype in COPD.
\end{abstract}

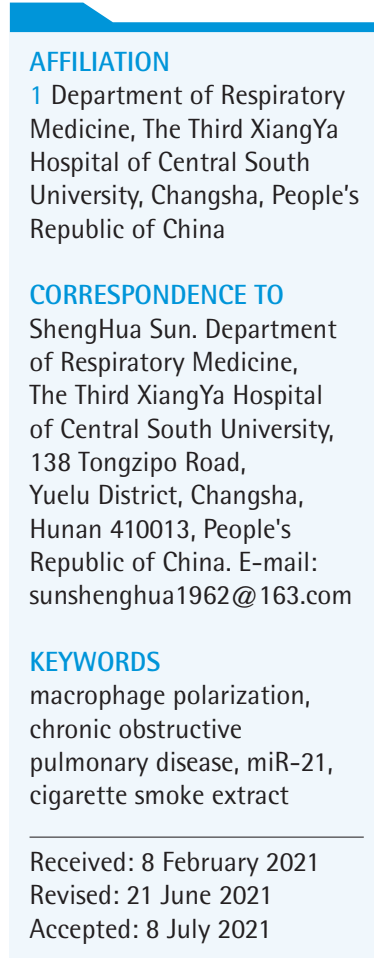

\section{INTRODUCTION}

COPD is one of the diseases with high economic burden and mortality in the world. It is estimated that more than 300 million people were affected by COPD in 2020 in the world, and 4.7 million of the 68 million deaths are caused by $\mathrm{COPD}^{1-3}$. The number of macrophages is increasing in the small airways of COPD patients ${ }^{4}$, and there is a close relationship between the number of macrophages and the severity of COPD ${ }^{4,5}$. Multiple studies have shown that the number of macrophages in the lung tissue of COPD patients is increased; in addition, there is a correlation between disease progression and the number of macrophages $^{5,6}$. Alveolar macrophages (AM) play an indispensable role in the occurrence and development of COPD, and, at the same time, it can affect the structure of lung tissue through the initiation and degeneration of inflammation ${ }^{7}$.

Mutative exogenous stimuli and pathological processes can change the phenotypes and functional characteristics of alveolar macrophages ${ }^{8}$. Since a large number of M1-type macrophage-related cytokines, such as TNF-a and IL-8, are found in bronchoalveolar lavage fluid (BAL), pulmonary macrophages from patients with COPD have been recommended to display M1 phenotypes ${ }^{9}$. In addition, TNF-a has been 
shown to be closely related to cigarette smokinginduced emphysema in mice ${ }^{10}$. Research has shown that CS can induce macrophages to reprogram to the M2 phenotype in patients with $\mathrm{COPD}^{11}$. In vitro, using different concentrations of CSE to interfere with macrophages, the expression of M2 macrophagerelated markers (CD163, CD204, and CD206) ) $^{12-14}$ and related factors (IL-10, TGF- $\beta 1$, TGF- $\beta 2$ ) was up-regulated ${ }^{15,16}$. Similarly, our previous studies showed that the proportion of M2 macrophages to M1 macrophages in BAL and lung tissue in a COPD mouse model were both significantly increased ${ }^{17,18}$. A number of etiology factors may contribute to changes in macrophage phenotypes including gramnegative anaerobe actinomycetes (AA), smoking and porphyromonas gingival (PG), and smoking is the most important factor ${ }^{19}$. However, the regulatory mechanism of macrophage polarization in COPD is unclear.

MicroRNAs (miRNAs) are small endogenous noncoding RNAs which bind in a sequence-specific manner to incomplete complementary sites in target messenger RNA (mRNA), thereby directly inhibiting protein translation or transcriptome degradation. In this way, miRNAs can simultaneously interact with hundreds of genes and regulate various developmental and physiological processes, including cell proliferation, differentiation, apoptosis, and innate and adaptive immune responses ${ }^{20}$. The research shows that miRNAs influence macrophage activation and polarization ${ }^{21}$. Our previous study confirmed that miR-21 was increased in lungs from a COPD mouse model and the peripheral blood of COPD patients, and its expression level correlated with lung function ${ }^{22}$. However, whether miR-21 is involved in COPD development by targeting macrophage polarization is unknown.

Therefore, in this study, we used CS exposure combined with intraperitoneal injection of CSE to construct miR-21 $1^{-/}$COPD animal models, to observe the effect of CSE on miR-21 and investigate the possible role of miR-21 in COPD-associated macrophage polarization.

\section{METHODS}

\section{Animals, preparation of CSE and animal model}

C57BL/6 and miR-21/-- C57BL/6(F1) mice were randomly divided into a control group and a COPD model group, all of which were purchased from the Jackson Laboratory. All animals were kept in clean rooms with a temperature of $23-25^{\circ} \mathrm{C}$, a humidity of $50-60 \%$ and a 12 -hour light/dark cycle. The study followed the guidelines for animal and human research ${ }^{23}$. The preparation of CSE followed previous procedure $^{24}$. The COPD mice model was established by CS exposure combined with intraperitoneal injection of CSE. The whole experiment lasted 28 days. The modeling box was made as previously described ${ }^{25}$. First, five cigarettes were lit simultaneously for 15 minutes. Second, the box is opened and the animals allowed to rest for five minutes. Then, the first step is repeat. The entire process is called CS exposure cycle, with exposure twice a day during the entire experimental cycle, except for the 1st, the 12th, and 23rd day; the control group was kept in the experimental animal center of the Third Xiangya Hospital of Central South University. The intraperitoneal injection of CSE was carried out according to the previous method. On days 1, 12, and 23 , the control animals were intraperitoneally injected with $0.3 \mathrm{~mL} / 20 \mathrm{~g}$ PBS, while the model animals were intraperitoneally injected with $0.3 \mathrm{~mL} / 20 \mathrm{~g}$ CSEPBS. On day 29, the lung function of the mice was measured, and lung tissue and BMDMs were collected. This research was approved by the Institutional Review Board of Central-South University and followed the guidelines for animal and human research ${ }^{23}$.

\section{Cell culture and production of BMDMs}

RAW264.7 and L929 cell lines were purchased from the American Type Culture Collection and were cultured using DMEM (Life Technologies, Carlsbad, CA, USA) containing $10 \%(\mathrm{v} / \mathrm{v})$ heat-inactivated FBS. According to our previous experiments, CSE interferes with RAW264.7 cells at a concentration of $5 \%$. BMDMs are established as previously described ${ }^{26}$.

\section{Histomorphology of lung tissue}

The left lower lung was lavaged with $4 \%$ paraformaldehyde, and then fixed with $4 \%$ paraformaldehyde overnight ${ }^{27}$. The lung tissue was embedded in paraffin (Sigma, MO, USA), cut it into $4 \mu \mathrm{m}$ sections after embedding, and stained with hematoxylin and eosin ( $\mathrm{H} \& \mathrm{E}$ ) (Sigma). The three indicators of mean linear intercept (MLI), mean alveolar interval thickness (MAST) and destruction 
index (DI) were used to quantify the pathological severity of emphysema. The measurement methods have been previously described ${ }^{25}$.

\section{Immunohistochemical staining}

The dewaxing and descaling sections were incubated with $1 \% \mathrm{H}_{2} \mathrm{O}_{2}$ at room temperature for 30 minutes to eliminate endogenous peroxidase activity, and then anti-CD68, anti-CD206 and anti-CD86 antibodies (ProInTech, Chicago, IL, USA) were added and incubated overnight at $4^{\circ} \mathrm{C}$. The sections were washed thoroughly on the second day and incubated with the secondary antibody for 1 hour at room temperature. The secondary antibody needs to be coupled with appropriate horseradish peroxidase. The unreacted secondary antibody was removed and the section was incubated with 3,39-diaminobiphenyl-4HCl (DAB; Sigma, MO, USA) $-\mathrm{H}_{2} \mathrm{O}_{2}$ solution to observe the immunolabeling, and then $\mathrm{HE}$ was used to counterstain the section using a fixed glass cover. The presence of CD68, CD206 and CD86 can be indicated by varying degrees of tan or brown particles or flake deposits. Four or five CD68, CD206 and CD86 positive images were randomly selected to calculate the integrated photometric value (IOD) and average optical density (AOD). Image-Pro Plus version 6.0 (Media Cybernetics, Inc., Rockville, MD, USA) software was used for high magnification of each slice to visualize high-definition color pathological images and analyze the results.

\section{Transient miRNA transfections}

The miRNA-21 inhibitor was purchased from Qiagen, and the all-star negative mimic (Qiagen) was used as a control. According to the transfection reagent instructions, the cells were transiently transfected with Hiperfect Transfection Reagent (Qiagen) at a final concentration of $50 \mathrm{nM}$. Red siGLO oligos (Thermo Fisher Scientific, Waltham, MA, USA) was used to confirm the transfection efficiency of the cells. The transfection efficiency was greater than $90 \%$ and the transfection was considered successful ${ }^{28}$.

\section{Quantitative real-time PCR}

According to the instructions, TRIzol reagent (Takara, Dalian, Liaoning, China) is used to isolate total RNA, and SYBR Green PCR Master Mix (Bio-Rad, CA, USA) used to detect mRNA levels. The mRNA expression was detected using a two-step quantitative real-time polymerase chain reaction (Applied Biosystems, Carlsbad, CA, USA). As endogenous control $\beta$-actin was used, and the DD cycle threshold method was used to determine the relevant expression level. Table 1 identifies the primer sequences.

\section{Statistical analysis}

The results were described using mean \pm standard deviation, and GraphPad Prism Software 6.0 (GraphPad Software, La Jolla, CA, USA) was used for data analysis. One-way analysis of variance was used for statistical evaluation, and the correlation between groups was analyzed using the Pearson correlation coefficient. A $p<0.05$ was considered statistically significant.

\section{RESULTS}

The expression of miR-21 is up-regulated in RAW264.7 cells intervened by CSE and lung tissue and BMDMs from COPD mouse model.

Our experiment detected the expression of miR21 in RAW264.7 cells intervened by CSE and lung tissue and BMDMs from COPD mouse model and miR-21/- mice using RT-PCR. The RAW264.7 cells

Table 1. Primer sequences of the genes investigated in RT-PCR analysis

\begin{tabular}{|c|c|}
\hline Gene & Primer sequences \\
\hline \multirow[t]{2}{*}{$\beta$-Actin } & F 5'-AACGGCTCCGGCATGTGCAA-3' \\
\hline & R 5'-CTTCTGACCCATGCCCACCA-3' \\
\hline \multirow[t]{2}{*}{ TNF-a } & F 5'-CAGCCTCтTСTCСтTCCTGAT-3' \\
\hline & R 5'-GCCAGAGGGCTGATTAGAGA-3' \\
\hline \multirow[t]{2}{*}{ IL-10 } & F5'-ACCAAGACCCAGACATCA-3' \\
\hline & R 5'-TTCACAGGGAAGAAATCG-3' \\
\hline \multirow[t]{2}{*}{ IL-6 } & F 5'-GGAACTCTACCAGAAATATAGC-3' \\
\hline & R 5'-CCTGTATTCCGTCTCCTG-3' \\
\hline \multirow[t]{2}{*}{ CD206 } & F 5'-GTGGAGTGATGGAACCCCAG-3' \\
\hline & R 5'-CTGTCCGCCCAGTATCCATC-3' \\
\hline \multirow[t]{2}{*}{ Ym1 } & F 5'-GAAGGAGCCACTGAGGTCTG-3' \\
\hline & R 5'-TGாGTCCTTGAGCCACTGA-3' \\
\hline \multirow[t]{2}{*}{ Fizz1 } & F 5'-CGTGGAGAATAAGGTCAAGGA-3' \\
\hline & R 5'-CAGTAGCAGTCATCCCAGCA-3' \\
\hline \multirow[t]{2}{*}{ ARG1 } & F 5'-GCATATCTGCCAAAGACATCG-3' \\
\hline & R 5'-CCATCACCTTGCCAATCCC-3' \\
\hline \multirow[t]{2}{*}{ iNOS } & F 5'- AGGGAATCTTGGAGCGAGTT-3' \\
\hline & R 5'- GCAGCCTCTTGTCTTTGACC-3' \\
\hline
\end{tabular}


Figure 1. The expression of miR-21 is up-regulated by cigarette smoke extracts. A: the expression level of miR-21 in lung tissue. Control group: normal control group. Model group: C57BL/6 mice were intervened by CS exposure combined with CSE intraperitoneal injection. The miR-21 $1^{-/}$model group: miR-21 $1^{-/} \mathrm{C} 57 \mathrm{BL} / 6$ mice were intervened by CS exposure combined with CSE intraperitoneal injection. $* p<0.05$, versus the control group; $* \& p<0.05$, versus COPD model group. B: the expression level of miR-21 in BMDMs.*p<0.05, versus the control group; $* \& p<0.05$, versus COPD mouse model. C: the expression level of miR-21 in RAW264.7 cells treated with CSE. *p<0.05, versus the control group. ${ }^{*} \& p<0.05$, versus the CSE-24h group.
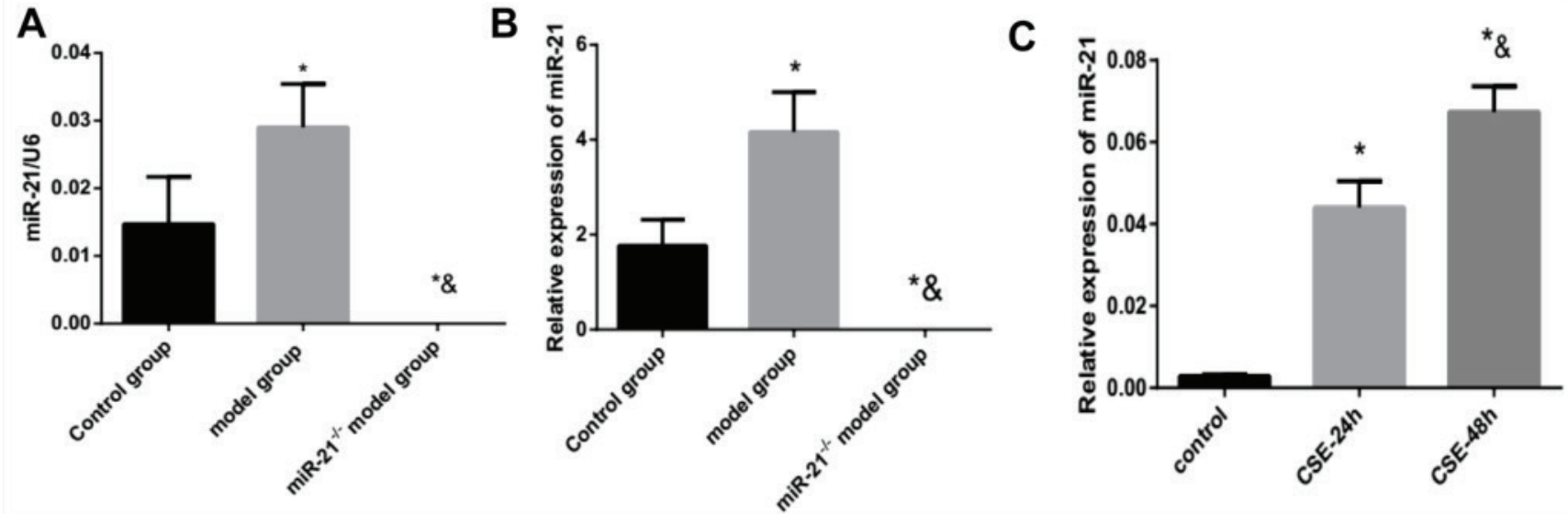

Figure 2. The change in M2/M1 macrophages and its correlation with miR-21. Control group: normal control group. Model group: C57BL/6 mice were intervened by CS exposure combined with CSE intraperitoneal injection. A: the protein expression levels of CD68, CD86 and CD206 as well as immunohistochemical staining for CD206 expression in lung tissue from COPD mouse model showing increased deposition of brown granules in cell membranes (black arrows, 400×magnification). B: ratio of M2/M1 macrophages. *p<0.05, versus the control group. C: ratio of M2 /M1 macrophages and its correlation with miR-21.

CD68

A
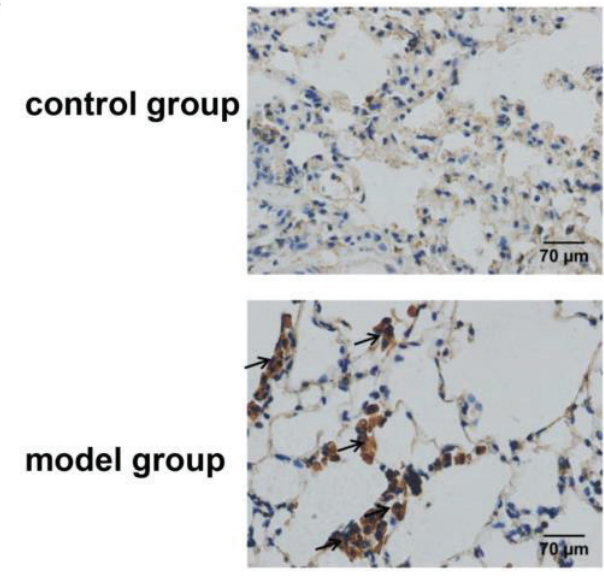

B

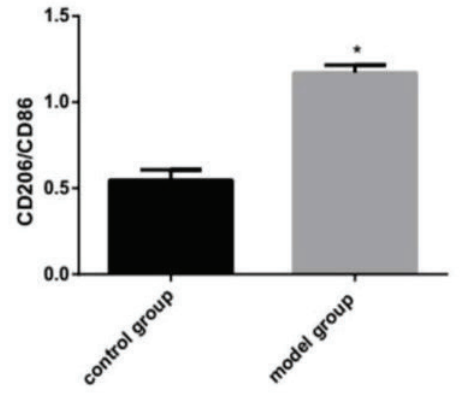

CD86
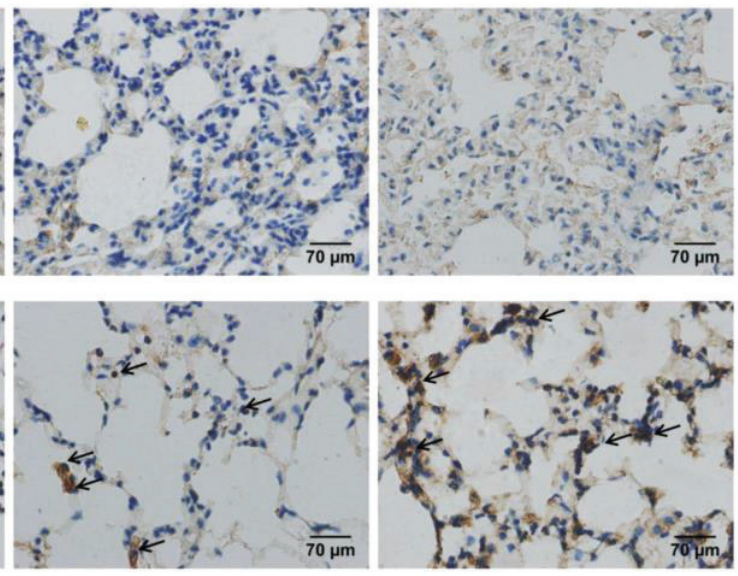

C

CD206

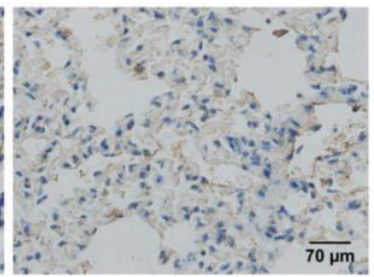

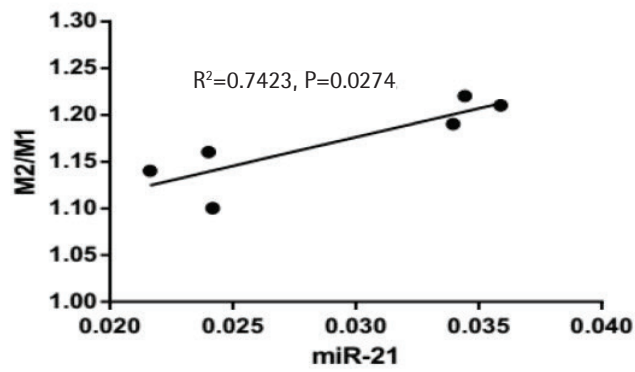


Figure 3. The pathological morphological changes of lung tissue after knocking out miR-21 while using the same method to build the COPD model. Model group: C57BL/6 mice were intervened by CS exposure combined with CSE intraperitoneal injection. The miR-21 $1^{-/}$group: miR-21 $1^{-/}$C57BL/6 mice were intervened by CS exposure combined with CSE intraperitoneal injection. A: HE staining of lung tissue from model mice (400×magnification) showing an enlarged alveolar space, a thinner alveolar septum and a destroyed alveolar wall (black arrows). B: HE staining of lung tissue from miR-21-/- mice (400×magnification) showing increased alveolar septum thickness, reduced alveolar size and destruction of the alveolar wall. C: comparison of lung histomorphology in the model group and the miR-21-/-group. MLI (um): mean linear intercept. MAST ( $\mu \mathrm{m})$ : mean alveolar septal thickness. DI (\%): destruction index. ${ }^{*} p<0.05$, versus the model group.

A

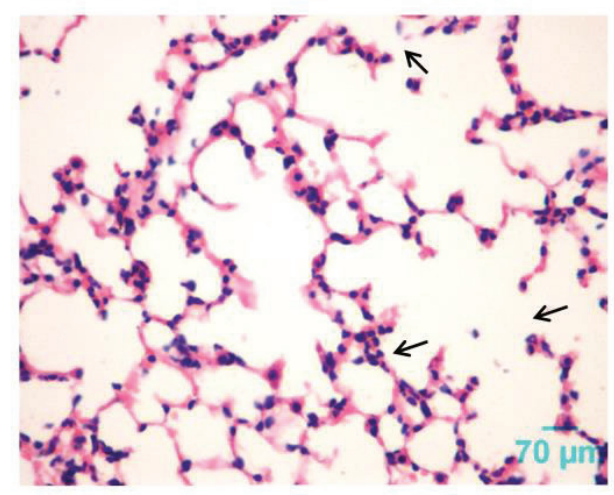

C

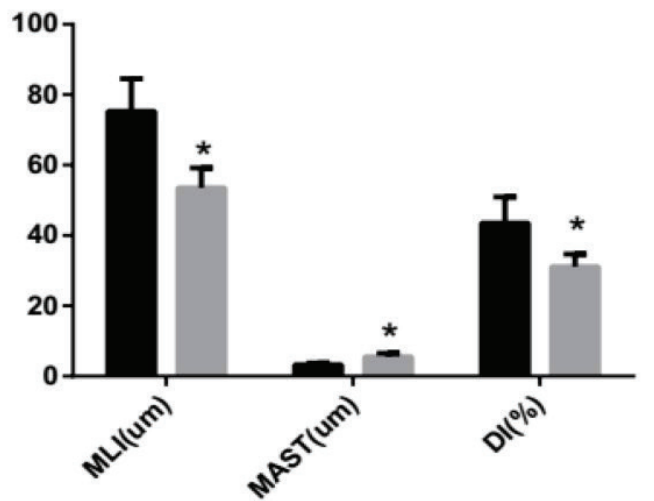

B

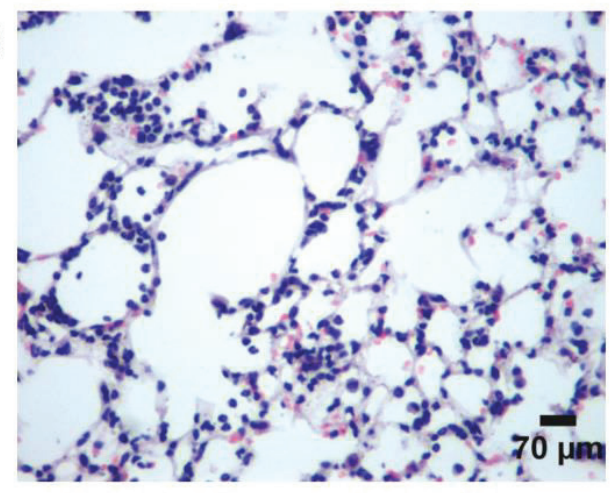

model group were intervened by $5 \% \mathrm{CSE}$ for $24 \mathrm{~h}$ and $48 \mathrm{~h}$ in vitro. Compared with the control group, the expression of miR-21 in lung tissues and BMDMs of COPD mice was significantly increased $(\mathrm{p}<0.05$, Figures $1 \mathrm{~A}$ and $1 \mathrm{~B})$. Moreover, CSE stimulation increased the expression of miR-21, which gradually rose over time $(\mathrm{p}<0.05$, Figure 1C).

The change in M2/M1 macrophages and its correlation with miR-21

In this experiment, immunohistochemical staining showed a rising proportion of M2 macrophages to M1 macrophages, which indicated the macrophages in the lung tissue of COPD mice had a tendency to polarize towards M2 macrophages $(\mathrm{p}<0.05$, Figures $2 \mathrm{~A}$ and
2B). Furthermore, the proportion of M2 macrophages to M1 macrophages was positively correlated with the expression of miR-21 $(\mathrm{p}<0.05$, Figure $2 \mathrm{C})$. In our COPD mice induced by the combination of CS exposure and intraperitoneal injection of CSE, macrophages were more likely to have the M2 phenotype.

The pathological morphological changes in lung tissue after knocking out miR-21

Using the same way to build the COPD mouse model, the alveolar septum thickness was increased compared to wild-type mice, and the alveolar size and destruction of the alveolar wall were reduced in miR21 knockout mice (Figures 3A and 3B). MLI and DI 
Figure 4. RNA expression levels of M1/M2 macrophages-related cytokines and markers in BMDMs from the control, COPD and miR-21 ${ }^{-/}$COPD groups. Control group: normal control group. Model group: C57BL/6 mice were intervened by CS exposure combined with CSE intraperitoneal injection. The miR-21 ${ }^{-1-}$ model group: miR-21 ${ }^{-/}$C57BL/6 mice were intervened by CS exposure combined with CSE intraperitoneal injection. $* p<0.05$, versus the control group. $\# p<0.05$, versus the model group.
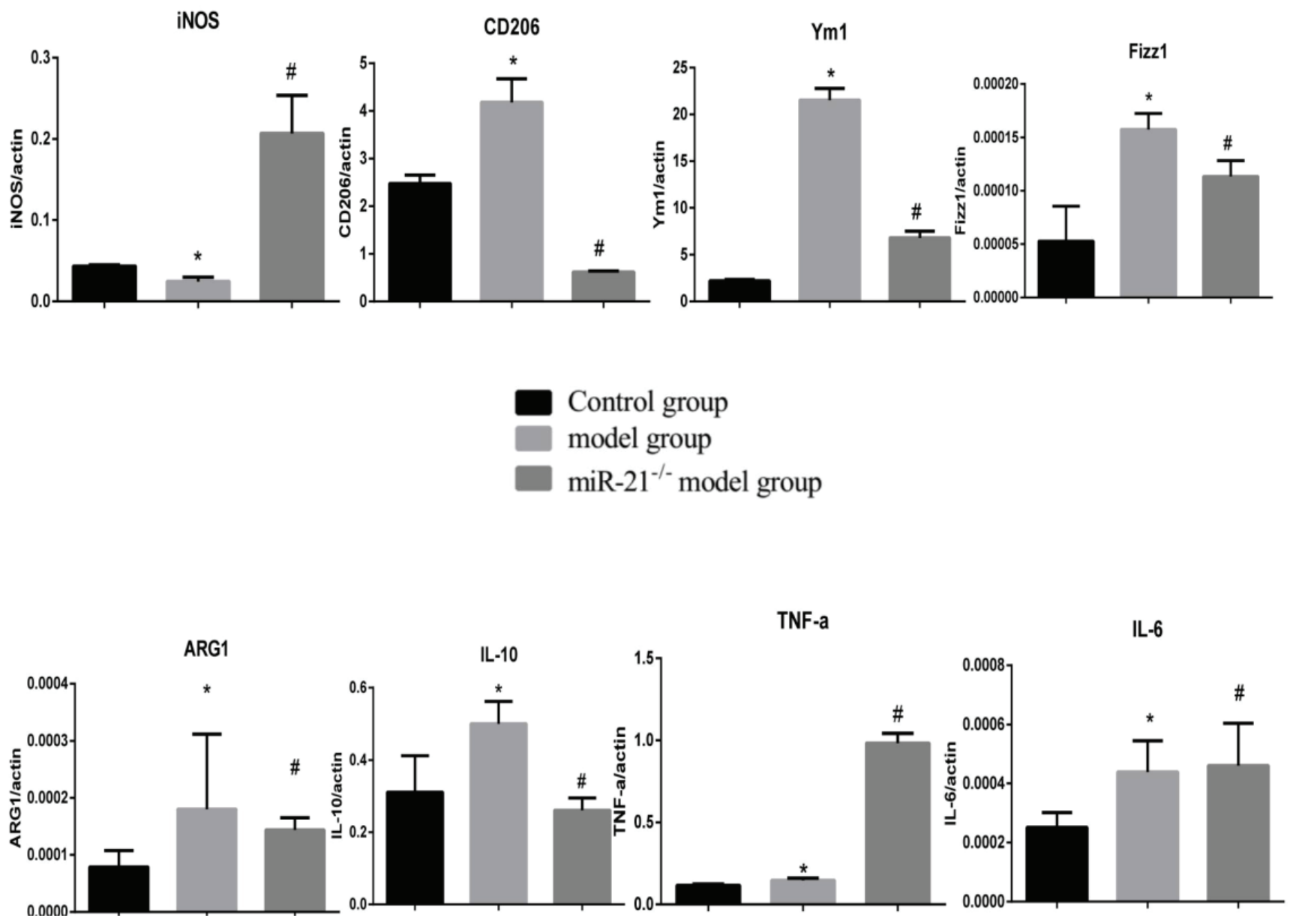

were reduced, and MAST was increased in miR-21 knockout mice $(\mathrm{p}<0.05$, Figure $3 \mathrm{C})$.

The effect of CSE and miR-21 on macrophage polarization in BMDMs and RAW264.7 cells

To clarify the effects of CSE and miR-21 on the polarization of macrophages, we used RT-PCR to detect the expression levels of inflammatory factors and markers associated with M1 and M2 macrophages (Table 1). Inducible nitric oxide synthase (iNOS), IL-6 and TNF-a were representative markers for M1 macrophages, and CD206, arginase 1 (ARG1), chitinase-like secretory lectin Ym1, resistin-like-a (Fizz1) and IL-10 were used as representative markers of M2 macrophages. Results suggested, compared with the control group, in BMDMs of COPD mice and RAW264.7 cells intervened by CSE, the mRNA expression levels of inflammatory factors and markers associated with M2 macrophages (CD206, Arg1, YM1, FizZ1, and IL-10) were increased $(\mathrm{p}<0.05$, Figures 4 and 5), and the expression of iNOS was decreased $(p<0.05$, Figures 4 and 5$)$. In the BMDMs from the miR-21 $1^{-/}$model group, the mRNA expression levels of inflammatory factors and markers associated with M2 macrophages (CD206, Arg1, YM1, FizZ1, and IL10) decreased ( $<<0.05$, Figure 4$)$, whereas the RNA expression level of inflammatory factors and markers associated with M1 macrophages (iNOS and TNF-a) increased $(p<0.05$, Figure 4B). However, there is no change in expression level of IL-6 ( $>>0.05$, Figure 
Figure 5. RNA expression levels of M1/M2 macrophages-related cytokines and markers in RAW264.7 cells from the control, cigarette smoke extract (CSE)-treated and miR-21 inhibitor-treated groups. CSE: 5\% CSE interfering with RAW264.7 cells. CSE+ miR-21 inhibitor: miR-21 inhibitor interfering with CSE-treated RAW264.7 cells. $* p<0.05$, versus the control group. $\# p<0.05$, versus the CSE group.
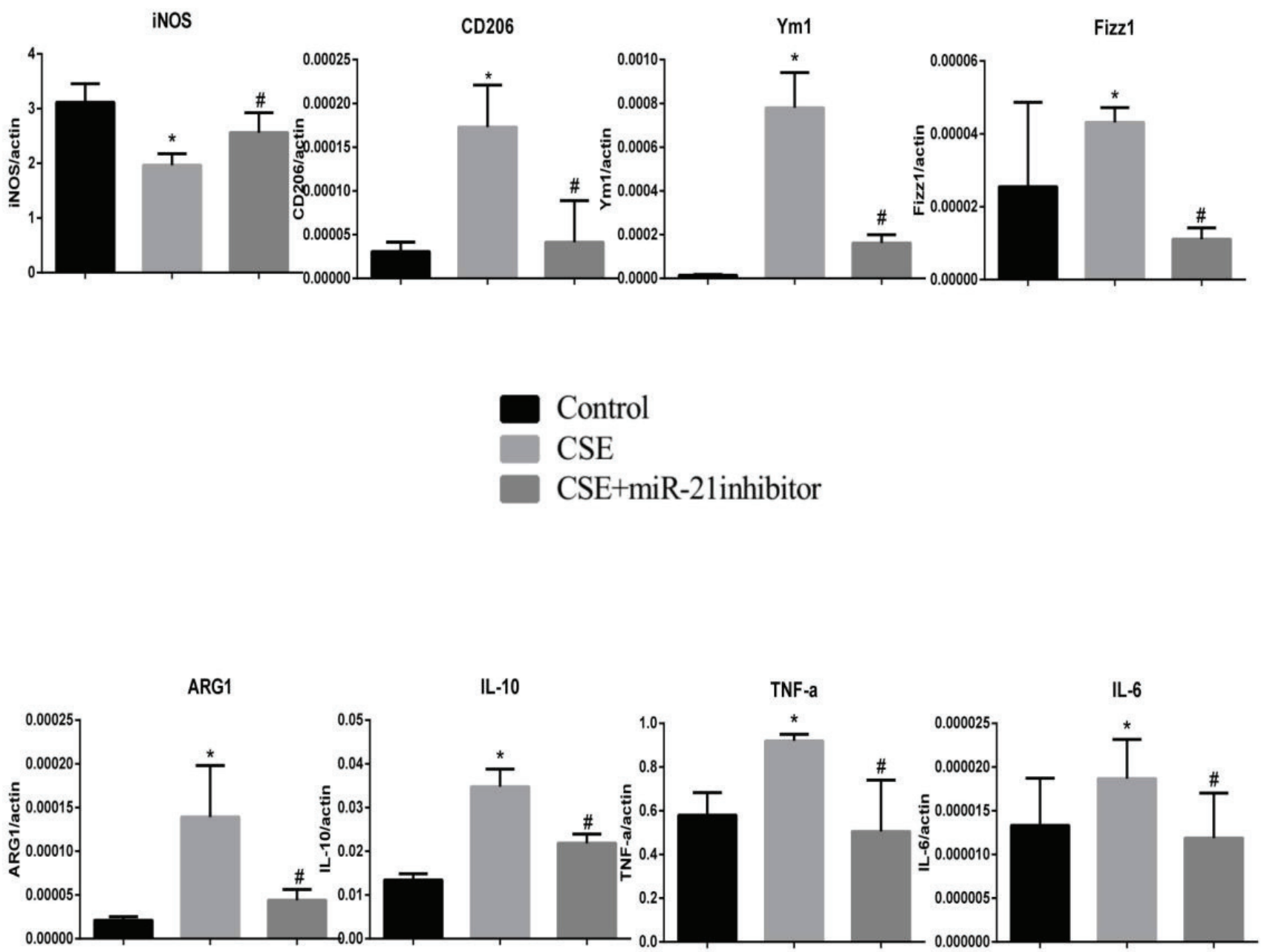

4). Adding miR-21 inhibitor to the RAW264.7 cells intervened by CSE reduced the changes of RNA expression levels of CD206, Arg1, YM1, FizZ1 and IL-10 ( $<<0.05$, Figure 5), while the RNA expression levels of iNOS, IL-10 and IL- 6 were increased $(\mathrm{p}<0.05$, Figure 5$)$.

\section{DISCUSSION}

We discovered the miR-21 expression was increased in RAW264.7 cells intervened by CSE and gradually increased with the CSE-intervention duration. At the same time, compared with the control group, the expression of miR-21 in BDMDs cells from COPD mice was significantly increased, which was basically consistent with our previous experimental results ${ }^{22}$.
As an important regulator, miRNAs exert a fine regulatory function on specific cellular events in the development of various organs, including the lungs. Our previous study also confirmed the differential expression of miRNAs in lung tissues of COPD rats and may have an important impact on the occurrence and development of $\mathrm{COPD}^{22,29}$. At the same time, our experiments suggested miR-21 is closely related to pathological changes in lung tissue. When our miR$21^{-/-}$mice were modelled using the same method as the method for wild-type mice, we found that compared with wild-type mice, miR-2 $1^{-/-}$mice have significantly reduced alveolar damage, which suggests miR-21 may have an important impact on the formation of emphysema and knockout miR-21 
can prevent the occurrence of emphysema as well as provide a new targeted therapy for the treatment of COPD.

Research has suggested CD68+ macrophages were increased in bronchial mucosa from COPD patients with mild/moderate stability compared with a control group $^{30}$. Previously, the expression of CD68+ cells in the small airways was inaccurate, but now studies have shown that compared with smokers with normal lung function, the number of CD68+ macrophages in small airways increases with the severity of $\mathrm{COPD}^{31}$. Our experiments also verified that the protein expression of CD68 expression was increased in the COPD mouse model. It is currently unclear whether M1 or M2 macrophages are dominant in COPD, and it is possible that an intermediate phenotype exists ${ }^{32}$. Our previous study showed that macrophage polarization occurred in bronchoalveolar lavage fluid in COPD mice and that there was a tendency towards M2 macrophages, which were closely associated with lung function and lung tissue destruction ${ }^{17}$. Our experiment also confirmed that compared with the control group, the ratio of CD206/CD86 is significantly increased, which was consistent with our previous results ${ }^{18}$; by adopting the two continuous section-contrasted staining method we confirmed that the proportion of M2 macrophages to M1 macrophages increased in the lung tissue from COPD mouse model ${ }^{18}$

MiRNAs may have an important influence and effect on the maturation of monocytes/macrophages. Our experiments verified that miR-2 1 inhibitors could decrease the expression of related inflammatory factors and markers associated with M2 macrophages in RAW264.7 cells with a CSE intervention. We found that M2 macrophage-related markers and factor expression were reduced in miR-21 knockout mice BDMDs cells compared with wild-type model mice, which indicated that miR-21 can mediate macrophage phenotype changes. Consistent with our observations, miR-21 is the central site for the mediation of macrophage phenotype changes from pro-inflammatory (M1) to pro-apoptotic (M2) via phagocytosis (phagocytotic apoptotic cells) ${ }^{33}$.

We also found that in different intervention environments, the expression of TNF-a, IL-6 and IL10 may be different, which indicates that CSE-induced macrophage transformation may exist between M1 and M2 dynamic changes. Additionally, inflammatory cytokines and immunosuppressive factors coexist, which is a condition that results in a low but persistent inflammation and leads to COPD, and regulation of macrophage polarization can be used as a treatment for COPD patients ${ }^{34}$. Our experiment also confirmed this paradox, which indicates that miR-21 may exert a different effect at different stages of COPD and may lead to the coexistence of a variety of macrophage types. This requires further study to discover the role of this dynamic change in COPD. Studies have shown that pri-miR-21 may have an effect in the early stages of inflammation, while mature miR-21 has an effect on inflammation repair ${ }^{35}$. At an early stage of inflammation, pri-miR-21 is predominant and exhibits proinflammatory effects. In contrast, during the inflammatory repair stage, mature miR-21 exerts an anti-inflammatory effect and also causes macrophages to transform into M2 macrophages, which exhibits a low inflammation level and an immunosuppressive state and leads to the continuation of inflammation. Whether this phenomenon exists in COPD requires further study. Moreover, our previous study establishing a dynamic COPD rat model showed that miR-21 expression was a dynamic process.

\section{Limitations}

Our experiment has some limitations. First, the mechanism of miR-21 on macrophage polarization was not studied in depth. Second, we have not studied the specific role of miR-21 in COPD, for example, epithelial-mesenchymal transition, endothelialmesenchymal transition, and small airway remodeling etc. We thus need to further study the mechanism and the function of miR-21 and M2 macrophages in COPD.

\section{CONCLUSIONS}

CSE can lead to macrophage transformation to the M2 phenotype and a rise in the expression of miR21. Knockout of the miR-21 gene may reduce lung injury in COPD mouse model and could inhibit the transformation of macrophages to the M2 phenotype in COPD.

\section{REFERENCES}

1. Vogelmeier CF, Criner GJ, Martínez FJ, et al. Global Strategy for the Diagnosis, Management, and Prevention of Chronic Obstructive Lung Disease 2017 Report: GOLD 
Executive Summary. In Spanish. Arch Bronconeumol. 2017;53(3):128-149. doi:10.1016/j.arbres.2017.02.001

2. Negewo NA, Gibson PG, Wark PA, Simpson JL, McDonald VM. Treatment burden, clinical outcomes, and comorbidities in COPD: an examination of the utility of medication regimen complexity index in COPD. Int J Chron Obstruct Pulmon Dis. 2017;12:2929-2942. doi:10.2147/COPD.S136256

3. GBD 2013 Mortality and Causes of Death Collaborators. Global, regional, and national age-sex specific all-cause and cause-specific mortality for 240 causes of death, 1990-2013: a systematic analysis for the Global Burden of Disease Study 2013. Lancet. 2015;385(9963):117-171. doi:10.1016/s0140-6736(14)61682-2

4. Di Stefano A, Capelli A, Lusuardi M, et al. Severity of airflow limitation is associated with severity of airway inflammation in smokers. Am J Respir Crit Care Med. 1998;158(4):12771285. doi:10.1164/ajrccm.158.4.9802078

5. Hogg JC, Paré PD, Hackett TL. The Contribution of Small Airway Obstruction to the Pathogenesis of Chronic Obstructive Pulmonary Disease. Physiol Rev. 2017;97(2):529-552. doi:10.1152/physrev.00025.2015

6. Mortaz E, Folkerts G, Redegeld F. Mast cells and COPD. Pulm Pharmacol Ther. 2011;24(4):367-372. doi:10.1016/j.pupt.2011.03.007

7. Vlahos R, Bozinovski S. Role of alveolar macrophages in chronic obstructive pulmonary disease. Front Immunol. 2014;5:435. doi:10.3389/fimmu.2014.00435

8. Gordon S, Plüddemann A, Martinez Estrada F. Macrophage heterogeneity in tissues: phenotypic diversity and functions. Immunol Rev. 2014;262(1):3655. doi:10.1111/imr.12223

9. Tangedal S, Aanerud M, Persson LJ, Brokstad KA, Bakke PS, Eagan TM. Comparison of inflammatory markers in induced and spontaneous sputum in a cohort of COPD patients. Respir Res. 2014;15(1):138. doi:10.1186/s12931-014-0138-6

10. Churg A, Wang RD, Tai H, Wang X, Xie C, Wright JL. Tumor necrosis factor-alpha drives $70 \%$ of cigarette smoke-induced emphysema in the mouse. Am J Respir Crit Care Med. 2004;170(5):492-498. doi:10.1164/rccm.200404-5110C

11. Shaykhiev R, Krause A, Salit J, et al. Smoking-dependent reprogramming of alveolar macrophage polarization: implication for pathogenesis of chronic obstructive pulmonary disease. J Immunol. 2009;183(4):2867-2883. doi:10.4049/jimmunol.0900473

12. Kunz LI, Lapperre TS, Snoeck-Stroband JB, et al. Smoking status and anti-inflammatory macrophages in bronchoalveolar lavage and induced sputum in COPD. Respir Res. 2011;12(1). doi:10.1186/1465-9921-12-34

13. Kaku Y, Imaoka H, Morimatsu Y, et al. Overexpression of CD163, CD204 and CD206 on alveolar macrophages in the lungs of patients with severe chronic obstructive pulmonary disease. PLoS One. 2014;9(1):e87400. doi:10.1371/journal.pone.0087400

14. Bazzan E, Turato G, Tinè M, et al. Dual polarization of human alveolar macrophages progressively increases with smoking and COPD severity. Respir Res. 2017;18(1):40. doi:10.1186/s12931-017-0522-0

15. Yuan F, Fu X, Shi H, Chen G, Dong P, Zhang W. Induction of murine macrophage M2 polarization by cigarette smoke extract via the JAK2/STAT3 pathway. PLoS One. 2014;9(9):e107063. doi:10.1371/journal.pone.0107063

16. Feng H, Yin Y, Ren Y, et al. Effect of CSE on M1/M2 polarization in alveolar and peritoneal macrophages at different concentrations and exposure in vitro. In Vitro Cell Dev Biol Anim. 2020;56(2):154-164. doi:10.1007/s11626-019-00426-4

17. Lu J, Xie L, Liu C, Zhang Q, Sun S. PTEN/PI3k/AKT Regulates Macrophage Polarization in Emphysematous mice. Scand J Immunol. 2017;85(6):395-405. doi:10.1111/sji.12545

18. He S, Xie L, Lu J, Sun S. Characteristics and potential role of M2 macrophages in COPD. Int J Chron Obstruct Pulmon Dis. 2017;12:3029-3039. doi:10.2147/COPD.S147144

19. Tonetti MS. Cigarette smoking and periodontal diseases: etiology and management of disease. Ann Periodontol. 1998;3(1):88-101. doi:10.1902/annals.1998.3.1.88

20. Bartel DP. MicroRNAs: genomics, biogenesis, mechanism, and function. Cell. 2004;116(2):281-297. doi:10.1016/s0092-8674(04)00045-5

21. Ponomarev ED, Veremeyko T, Weiner HL. MicroRNAs are universal regulators of differentiation, activation, and polarization of microglia and macrophages in normal and diseased CNS. Glia. 2013;61(1):91-103. doi:10.1002/glia.22363

22. Xie L, Wu M, Lin H, et al. An increased ratio of serum miR-21 to miR-181a levels is associated with the early pathogenic process of chronic obstructive pulmonary disease in asymptomatic heavy smokers. Mol Biosyst. 2014;10(5):1072-1081. doi:10.1039/c3mb70564a

23. World Medical Association, American Physiological Society. Guiding principles for research involving animals and human beings. Am J Physiol Regul Integr Comp Physiol. 2002;283(2):R281-R283. doi:10.1152/ajpregu.00279.2002

24. Chen Y, Hanaoka M, Droma Y, Chen P, Voelkel NF, Kubo K. Endothelin-1 receptor antagonists prevent the development of pulmonary emphysema in rats. Eur Respir J. 2010;35(4):904-912. doi:10.1183/09031936.00003909

25. He ZH, Chen P, Chen Y, et al. Comparison between cigarette smoke-induced emphysema and cigarette smoke extract-induced emphysema. Tob Induc Dis. 2015;13(March). doi:10.1186/s12971-015-0033-z

26. Sutterwala FS, Noel GJ, Clynes R, Mosser DM. Selective suppression of interleukin-12 induction after macrophage receptor ligation. J Exp Med. 1997;185(11):1977-1985. doi:10.1084/jem.185.11.1977

27. Yao H, Chung S, Hwang JW, et al. SIRT1 protects against emphysema via FOXO3-mediated reduction of premature senescence in mice. J Clin Invest. 2012;122(6):2032- 
2045. doi:10.1172/JCI60132

28. Fordham JB, Naqvi AR, Nares S. Regulation of miR-24, miR$30 \mathrm{~b}$, and miR-142-3p during macrophage and dendritic cell differentiation potentiates innate immunity. J Leukoc Biol. 2015;98(2):195-207. doi:10.1189/jlb.1A1014-519RR

29. Zhang R, Sun S, Ji F, et al. CNTN-1 Enhances Chemoresistance in Human Lung Adenocarcinoma Through Induction of Epithelial-Mesenchymal Transition by Targeting the PI3K/Akt Pathway. Cell Physiol Biochem. 2017;43(2):465-480. doi:10.1159/000480473

30. Postma DS, Reddel HK, ten Hacken NH, van den Berge M. Asthma and chronic obstructive pulmonary disease: similarities and differences. Clin Chest Med. 2014;35(1):143-156. doi:10.1016/j.ccm.2013.09.010

31. Hogg JC. Pathophysiology of airflow limitation in chronic obstructive pulmonary disease. Lancet. 2004;364(9435):709721. doi:10.1016/S0140-6736(04)16900-6

32. Belchamber KBR, Donnelly LE. Macrophage Dysfunction in Respiratory Disease. Results Probl Cell Differ. 2017;62:299-313. doi:10.1007/978-3-319-54090-0_12

33. Das A, Ganesh K, Khanna S, Sen CK, Roy S. Engulfment of apoptotic cells by macrophages: a role of microRNA-21 in the resolution of wound inflammation. J Immunol. 2014;192(3):1120-1129. doi:10.4049/jimmunol.1300613

34. Sun X, Liu Y, Feng X, Li C, Li S, Zhao Z. The key role of macrophage depolarization in the treatment of COPD with ergosterol both in vitro and in vivo. Int Immunopharmacol. 2020;79:106086. doi:10.1016/j.intimp.2019.106086

35. Kumarswamy R, Volkmann I, Thum T. Regulation and function of miRNA-21 in health and disease. RNA Biol. 2011;8(5):706-713. doi:10.4161/rna.8.5.16154
CONFLICTS OF INTEREST

The authors have completed and submitted the ICMJE Form for Disclosure of Potential Conflicts of Interest and none was reported.

\section{FUNDING}

This study was supported by grants from the National Natural Science Foundation of China (No. 81800044).

\section{ETHICAL APPROVAL AND INFORMED CONSENT}

This research was approved by the Institutional Review Board of Central-South University (No. 81800044, 2019.01-2021.12) and followed the guidelines for animal and human research.

\section{DATA AVAILABILITY}

The data supporting this research are available from the authors on reasonable request.

\section{AUTHORS' CONTRIBUTIONS}

JJL conceived the study, performed the experiment, data analysis, and drafted the manuscript. SHS coordinated the study. LHX guided the experiment. All authors read and approved the final manuscript.

PROVENANCE AND PEER REVIEW

Not commissioned; externally peer reviewed. 